\title{
LA TRANSICIÓN DE LA TEORÍA DEL EQUILIBRIO PUNTUADO HACIA UNA TEORÍA DE RANGO MEDIO
}

\author{
Vladimir Cachón y Ana Barahona
}

Facultad de Ciencias, UNAM

\section{RESUMEN}

Se analiza la teoría del equilibrio puntuado y las causas de que habiendo sido enunciada como una teoría evolutiva de amplio alcance en la actualidad sólo sea una teoría de aplicación limitada, o de rango medio. Se estudian los factores responsables de esta transformación y se concluye con una visión sobre lo que cabe esperar para el futuro próximo.

PALABRAS CLAVE: equilibrio puntuado, síntesis moderna, teorías de rango medio, registro fósil.

\section{SUMMARY}

The punctuated equilibrium theory is analysed as well as the causes of why at the present time it has become a middle-range theory although it was originally presented as a wide range evolution theory. The facts responsible for this transformation are studied. The article concludes with a vision of what is to be expected in the near future.

KEY WORDS: punctuated equilibrium, modern synthesis, middle-range theories, fossil record.

\section{INTRODUCCIÓN}

La teoría del equilibrio puntuado surgió en el año 1972 cuando Niles Eldredge y Stephen Jay Gould publicaron su primer artículo conjunto, intitulado «Punctuated equilibria: an alternative to phyletic gradualism» ${ }^{1}$. En este trabajo, Eldredge y Gould llamaban la atención sobre una contradicción existente entre las publicaciones paleontológicas tradicionales y la síntesis moderna: la evolución filética mostrada por los paleontólogos en sus reconstrucciones se oponía al modelo de especiación geográfica sostenido por Ernst Mayr y otros autores posteriores a la síntesis moderna.

1 Eldredge, N.; Gould, S.J. (1972), «Punctuated equilibria: an alternative to phyletic gradualism», en SCHOPF, T.J. (ed.), Models of Paleobiology, San Francisco, Freeman, Cooper \& Co., pp. 82-115. 
Según el modelo de especiación peripátrica que postula $\mathrm{Mayr}^{2}$, las nuevas especies surgen a partir de poblaciones locales periféricas, situadas al margen de la distribución geográfica central de la especie madre. Sin embargo, este modelo no deja lugar a la evolución progresiva in situ de las especies, que era precisamente lo que mostraban las reconstrucciones de especiación filética elaboradas hasta entonces por los paleontólogos.

Tanto Eldredge como Gould intentaron deshacer esta contradicción aplicando el modelo de especiación geográfica al registro fósil. Su conclusión fue que el gradualismo filético estaba, en el mejor de los casos, desmesuradamente sobrevalorado. La especiación se realizaba, más bien, mediante la transformación rápida de poblaciones periféricas. Además, este modelo proponía que, entre un evento y otro de especiación lo que hay son largos períodos de estasis, que aparecen puntuados -o interrumpidos-, por breves períodos de evolución rápida.

En síntesis, Eldredge y Gould propusieron que raramente hay gradualismo filético - como ellos rebautizaron a la anagénesis - sino que, durante la mayor parte del período de vida de una especie, lo que encontramos es una prolongada estasis morfológica puntuada, de vez en cuando, por eventos de especiación que ocurren, principalmente, en poblaciones marginales.

Esta teoría desató, desde su inicio, una gran polémica, sobre todo a partir de su posterior radicalización. En la actualidad la polémica ha disminuido en intensidad, pero sigue existiendo. Los autores puntuacionistas siguen muy activos en sus publicaciones $^{3}$ y recientemente han puesto el acento en un aspecto que, aunque jugó un papel secundario en los artículos iniciales sobre el equilibrio puntuado, ha ido cobrando cada vez mayor atención: la idea de que, además de la selección natural, existen fenómenos de selección al nivel de especies e incluso a otros niveles jerárquicos superiores, mediante mecanismos que no pueden ser entendidos sólo con las categorías explicativas del nivel jerárquico inferior.

Lo que en el presente artículo nos proponemos explorar es el porqué de una aparente paradoja: por qué razón si el equilibrio puntuado fue presentado — sobre todo a inicios de los ochenta - como una teoría que cuestionaba fuertemente a la síntesis moderna y que se proponía a sí misma como un modelo alternativo, es decir, como

2 MAYR, E. (1954), «Change of genetic environment and evolution», en HUXLEY, J. (ed.) Evolution as a Precess, Londres, Allen \& Unwin, pp. 157-180. MAYR, E. (1970), Populations, Species and Evolution, Cambridge, Harvard University Press.

3 Ver, por ejemplo, GOULD, S. J. (1992), «Punctuated Equilibrium in Fact and Theory», en SOMIT, A. y S. PETERSON (ed.), The Dynamics of Evolution. The Punctuated Equilibrium Debate in the Natural and Social Sciences, Ithaca, Cornell University Press, pp. 54-84; y el extenso capítulo 9 de GouLD, S.J. (2002), The Estructure of Evolutionary Theory, Cambridge, Harvard University Press, pp. 745-1024; también EldRedge, N. (1995), Reinventing Darwin: The Great Debate at the High Table of Evolutionary Theory, Nueva York, Jhon Wiley \& Sons, pp. 64-79; y ElDREDGE, N. (1999), The Pattern of Evolution, New York, Freeman \& Co., pp. 16-23; 140-145. 
una teoría capaz de explicar patrones universales - y no sólo casos particulares-, a treinta años de su publicación inicial ha logrado sobrevivir a pesar de nunca haber desplazado a la teoría síntética de la evolución.

Nuestra hipótesis es que el equilibrio puntuado ha sobrevivido hasta ahora gracias a que se ha convertido en una teoría de rango medio ${ }^{4}$. Estable, periférica, y casi absorbida por la propia teoría sintética de la evolución ${ }^{5}$ a la que pretendió sustituir. El equilibrio puntuado, en la actualidad, es aceptado como posible respuesta para algunos casos particulares de la evolución, mas no como un modelo que explique patrones generales.

Consideramos que de esta manera se explica tanto su supervivencia como la declinación de las refutaciones que actualmente recibe de la síntesis moderna. Ambos fenómenos quedan mejor comprendidos si aceptamos que el equilibrio puntuado es, actualmente, sólo una teoría de rango medio, cuyos planteamientos se consideran convenientemente asimilados ya por el cinturón protector de la síntesis moderna.

Para desarrollar nuestro argumento divideremos el análisis en cuatro partes. En la primera parte hacemos un breve repaso del origen del equilibrio puntuado y de su posterior radicalización. En la segunda, mostramos la evidencia de que la teoría ha sobrevivido hasta ahora. En la tercera, presentamos la argumentación a favor de nuestra hipótesis: que el equilibrio puntuado se ha convertido en la actualidad en una teoría de rango medio. Por último, en la cuarta parte presentamos las dificultades empíricas que han acompañado a esta teoría y que, a nuestro modo de ver, complementan la explicación sobre su situación actual.

\section{EL SURGIMIENTO DE LA TEORÍA DEL EQUILIBRIO PUNTUADO Y SU POSTERIOR RA- DICALIZACIÓN}

El equilibrio puntuado no ha sido una teoría estática. Por el contrario, ha presentado importantes cambios a lo largo de su existencia antes de derivar, en la actualidad, en una teoría de rango medio.

\section{La primera etapa del equilibrio puntuado}

En su primera etapa — que comienza con el artículo fundacional de 1972-, las propuestas de Eldredge y Gould fueron presentadas como un ajuste necesario que

4 El término teorías de rango medio fue propuesto originalmente por Robert Merton para definir aquellas teorías de aplicación particular a aspectos delimitados de los fenómenos, en contraposición a las teorías de aplicación general. Véase más adelante, en la tercera parte de este trabajo, una exposición más detallada de este término.

5 En este artículo emplearemos, indistintamente, los términos sintesis moderna y teoría sintética de la evolución para referirnos a la teoría actual de la evolución, forjada durante la década de los 40 del siglo XX. 
debía hacérsele a los estudios paleontológicos para que éstos fueran congruentes con el mecanismo de especiación peripátrica propuesto por Mayr. De este modo, cuando Eldredge y Gould sostuvieron que el registro fósil no muestra un cambio gradual de las especies, sino más bien una gran uniformidad de las mismas a lo largo del tiempo geológico, y que esta estasis estaba interrumpida por cambios evolutivos rápidos durante los cuales aparecían las nuevas especies, también argumentaron que la síntesis moderna predecía que mucho - si no es que la mayor parte- de los cambios debían ocurrir durante los momentos de especiación, que estos eventos de especiación eran alopátricos en su mayoría, y que el principio del fundador de Mayr ${ }^{6}$ desempeñaba un papel crucial.

Con este nuevo enfoque, Eldredge y Gould también pretendían cambiar la visión que, hasta entonces, se tenía sobre el registro fósil: de ser una imperfecta colección de algunos taxones debería ser visto, en lo sucesivo, como el fiel reflejo del mecanismo seguido por la evolución para el surgimiento de nuevas especies. Bajo esta moderna concepción del proceso de especiación el material empírico de la evolución, es decir el registro fósil, quedaba al fin bien entendido y, por lo tanto, ya no había necesidad de ninguna hipótesis auxiliar que sostuviera que este registro era incompleto. Por el contrario, Eldredge y Gould se manifestaban satisfechos con la información que proveía.

Sin embargo, con ninguna de las propuestas de este artículo pretendían sus autores enfrentarse a la síntesis moderna. Al contrario: lo que sostenían era que sólo aho$\mathrm{ra}$, gracias a este nuevo enfoque, se estaba aplicando correctamente a la paleontología el mecanismo de especiación que formaba parte de la teoría sintética de la evolución.

A pesar de esto, la polémica no se hizo esperar y la teoría del equilibrio puntuado fue cuestionada, desde su surgimiento, por los exponentes de la síntesis moderna. El primer aspecto que recibió atención en contra, fue la imagen contundente que Eldredge y Gould daban del patrón de la evolución. Mayr ${ }^{7}$ hace notar que ellos, si bien no mencionaban explícitamente en su artículo inicial que una especie recién formada entra en un período de estasis total, sí es precisamente eso lo que mostraban en las gráficas ${ }^{8}$ del artículo. Mas aún, las tendencias evolutivas eran presentadas como el resultado de un proceso de selección al nivel de especies entre entidades completamente estáticas.

Un segundo elemento que provocó reacciones contrarias, lo constituyó la presentación de la teoría como algo completamente novedoso. Gould y Eldredge ${ }^{9}$ han insistido en que ellos fueron los primeros en llamar la atención sobre varios fenómenos

\footnotetext{
6 MAYR (1954).

7 MAYR, E. (1992), «Speciational Evolution or Punctuated Equilibria» en SOMIT, A.; PETERSON, S. (ed.), The Dynamics of Evolution. The Punctuated Equilibrium Debate in the Natural and Social Sciences, Ithaca, Cornell University Press, pp. 21-53.

8 Se refiere a las figuras 5-4, 5-8 y 5-10 de ELDREDGE y GOULD (1972).

9 Gould, S. J. y N. EldREDGE (1977), «Punctuated equilibria: the tempo and mode of evolution reconsidered», Paleobiology, 3, pp. 115-151.
} 
evolutivos (diferentes tasas de cambio, aparición rápida de especies, estasis). Pero, para otros autores, eran fenómenos ya ampliamente aceptados en la literatura sobre evolución ${ }^{10}$. De manera particular se les señaló que el gradualismo en Darwin era una negación del saltacionismo, pero que no implicaba en modo alguno la noción — que Gould y Eldredge le adjudicaban - de que la evolución no podía tener diferentes tasas de cambio.

La tercera situación que despertó hostilidad fueron los reclamos acerca de que la síntesis moderna necesitaba ser revisada. Dawkins ${ }^{11}$, entre otros, les insistió, desde el inicio de la polémica, en que el modelo del equilibrio puntuado podía entrar perfectamente en la esfera gradualista de la teoría sintética de la evolución; no había necesidad de contar con procesos macroevolutivos particulares para explicar las transformaciones evolutivas, aunque éstas fueran «puntuadas».

La base empírica de la teoría

A pesar de la polémica, pronto se sumaron a la teoría del equilibrio puntuado otros importantes paleontólogos, como Steven Stanley ${ }^{12}$, Alan Cheetham ${ }^{13}$, P. G. Williamson ${ }^{14}$ y Donald Prothero ${ }^{15}$. Convencidos de que la teoría podría probarse empíricamente, se hicieron importantes estudios cuyos resultados parecían validar el nuevo esquema teórico.

Entre estos estudios resalta, en primer lugar, el elaborado por Eldredge ${ }^{16}$ con un linaje de trilobites del Devónico y que fue el que aportó la base empírica inicial para el artículo fundacional de la teoría.

Phacops rana, como los restantes miembros de la familia de los facopideos, posee unos ojos compuestos mucho más complejos que los de los trilobites de otras familias. Cada ojo se compone de múltiples lentes diminutas, dispuestas en franjas vertica-

\footnotetext{
10 Stebbins, G. L.; Ayala, F. J. (1981), «Is a new evolutionary synthesis necessary?», Science, 213, pp. 967-971.

11 DAWkins, R. (1996), The Blind Watchmaker, Londres, Norton \& Co., pp. 223-252.

12 Stanley, S. (1979), Macroevolution: Pattern and Process, San Francisco, Freeman; StANLEY, S. (1982a), «Speciation and the fossil record», en BARIGOZZI, C. (ed.), Mechanisms of Speciation, Nueva York, Alan R. Liss, pp. 41-50; STANLEY, S. (1982b), «Macroevolution and the fossil record», Evolution, 36, pp. 460-473.

13 Cheetham, A. H. (1986), «Tempo of evolution in a Neogen bryozoan: rates of morphological evolution within an across species boundaries», Paleobiology, 12, pp. 190-202.

14 Williamson, P. G. (1981), «Paleontological documentation of speciation in Cenozoic mollusks from Turkana Basin», Nature, 292, pp. 437-443.

15 Prothero, D. (1992), «Punctuated Equilibrium at Twenty: a Paleontological Perspective», Skeptic, 1, pp. 38-47.

16 ELdREDGE, N. (1971), «The allopatric model and phylogeny in Paleozoic invertebrates», Evolution, 25, pp. 156-167.
} 
les. En el caso de $P$. rana fue la variación en el número de franjas verticales de lentes, en las poblaciones del Devónico medio de América del Norte, la que dio la clave que permitiría reinterpretar la evolución de la especie. En esta época, la mitad este de América del Norte se hallaba cubierta por un mar epicontinental poco profundo.

Los fósiles que permiten seguir la evolución de $P$. rana se escalonan a lo largo de ocho millones de años. Los trilobites se recogieron por una parte en los sedimentos depositados por el mar epicontinental — la zona de distribución principal (Michigan, Ohio, Ontario) - y, por otra parte, en la zona llamada marginal, un surco profundo excavado a lo largo del continente (Nueva York y más al Este). La sucesión de los trilobites en los depósitos del mar epicontinental muestra una reducción en el número de franjas de las lentes oculares, que pasan de 18 a 15: una población con 15 franjas sucedía a una población de 17 franjas, que a su vez sucedía a una población de 18 franjas. Las comparaciones con otros Phacops muestran que 18 franjas representa el estado primitivo. Ahora bien, las distintas poblaciones que se sucedían a lo largo de 8 millones de años en el mar epicontinental no manifestaban variaciones en el número de franjas. Estas variaciones sólo aparecían en las poblaciones de trilobites descubiertas en la zona marginal situada más al este. Más aún, las poblaciones dotadas de 17 franjas se habían individualizado allí rápidamente y mucho antes de su aparición en el amplio mar situado más al oeste. Durante más de dos millones de años los Phacops de 17 franjas habían sido contemporáneos de los Phacops de 18 franjas, pero vivían en otra parte.

El fenómeno se repitió después, de manera similar, en lo que respecta a las poblaciones marginales de 15 franjas. Estas poblaciones con menor número de lentes oculares sólo se dieron en zonas marginales producidas por una regresión marina. Posteriormente, las poblaciones de $P$. rana de 15 franjas fueron cercando la zona epicontinental y, aprovechando el retorno del mar, fueron sustituyendo a las poblaciones que vivían anteriormente en la zona. En la región epicontinental, la zona principal, no se produjo ninguna transformación gradual in situ. La especie $P$. rana resultaba ser una especie morfológicamente estable, en estasis, y los cambios evolutivos habían ocurrido únicamente - y de manera rápida - en las poblaciones marginales (conforme al modelo de especiación propuesto por Mayr), y no bajo el esquema de especiación filética (anagénesis).

Por su parte, Gould trabajó sobre todo con gasterópodos (en particular el género Cerion), pero también realizó la primera investigación con mamíferos cuyos resultados tienen que ver con el modelo del equilibrio puntuado: Ischyromys, un género de roedores del Oligoceno. Los Ischyromys del Orellense (la parte media del Oligoceno) del Oeste norteamericano habían sido objeto de una interpretación tradicional consecuente con el modelo gradualista convencional. La secuencia orellense había sido interpretada como la crónica de un incremento constante de tamaño en el seno de una sola especie. Sin embargo, el trabajo estadístico realizado por Gould y Heaton ${ }^{17}$ so-

\footnotetext{
17 Gould, S. J. (1994), Ocho cerditos, Barcelona, Crítica, pp. 263-265.
} 
bre varios miles de especímenes refutó esta idea en favor de una interpretación opuesta. En el Orellense inferior descubrieron dos especies distintas, una pequeña y otra mayor; después, la pequeña se extingue y sólo la de mayor tamaño perdura en el Orellense superior. Ninguna de las dos especies muestra modificaciones significativas durante su tiempo de existencia. La antigua idea sobre un incremento gradual de tamaño se debía, por tanto, al resultado de confundir ambas especies y tratarlas como si constituyeran una sola forma. A medida que la especie de menor tamaño decrece en abundancia (hasta llegar, finalmente, a desaparecer), la talla media del complejo entero va aumentando, pero no porque tenga lugar una evolución gradual por anagénesis, sino porque un número progresivamente mayor de especímenes (y finalmente todos) son representantes de la forma estable de mayor tamaño.

Pero ha sido P. G. Williamson ${ }^{18}$, del Museo de Zoología Comparada de la Universidad de Harvard, quien ha llevado a cabo el más detallado —y el más citado— de todos los estudios de campo que han aportado una base empírica a la teoría del equilibrio puntuado. Su estudio fue hecho con los moluscos de la cuenca del lago Turkana, en el noreste de Kenya, en una secuencia de estratos correspondientes al Cenozoico tardío. Esta secuencia resultó particularmente útil para su estudio ya que los taxones fósiles que contiene están inusualmente bien representados, son muy abundantes, pertenecen a grupos taxonómicos muy heterogéneos y, además, pertencen a linajes que aún tienen especies vivas, por lo que resulta posible hacer comparaciones e inferencias con ellas.

Williamson analizó y tomó medidas a miles de especímenes correspondientes a 13 linajes diferentes (entre ellos destacaron los prosobranquios Bellamya unicolor y Melanoides tuberculata). Sus resultados fueron los siguientes: el patrón filogenético de todos los moluscos estudiados en el lago Turkana resultaba consistente con el previsto por el modelo del equilibrio puntuado; períodos muy largos de estasis morfológica en todos los linajes estudiados aparecían puntuados por rápidos episodios de cambio fenotípico. En ningún linaje se apreciaba una tendencia gradual y constante de cambio morfológico. Además, en al menos dos casos, habían quedado claramente documentados los eventos de especiación ocurridos en poblaciones periféricas aisladas (como predice el principio del fundador de $\mathrm{Mayr}^{19}$ ).

De acuerdo al grosor de la capa estratigráfica que contiene el momento de cladogénesis, Williamson estimó que este evento de especiación debe haberse llevado a cabo en un período de entre 5.000 y 50.000 años. En todos los casos, y previo al momento de cladogénesis, Williamson encontró que las poblaciones periféricas entraban en una fase de elevación significativa de su varianza fenotípica, lo cual interpretó como un período de inestabilidad ontogenética, provocado por la alteración de los mecanismos de homeostasis génica que antecedería a los eventos de especiación.

18 WiLLIAMSON (1981).

19 MAYR (1954). 
La segunda etapa del equilibrio puntuado

Apenas cinco años después de su artículo inicial, ocurrió un cambio de actitud en los autores del equilibrio puntuado - cuyo punto cumbre podríamos situar con el artículo de Gould de $1980^{20}$ «Is a new and general theory of evolution emerging?»y, entre 1977 y 1982, adoptaron una versión radical de la teoría —que Hoffman ${ }^{21}$ ha llamado «la versión fuerte» y Ruse $^{22}$ «la segunda fase»— en la cual, para poder explicar su postulado macroevolutivo, propusieron que había una diferencia biológica fundamental entre los procesos que rigen la microevolución y los que rigen la especiación ${ }^{23}$. Para ello, invocaron nuevamente las tesis sobre especiación de Ernst Mayr así como las de Sewall Wright sobre deriva génica, en apoyo a su reclamo de que la especiación siempre debería ser muy rápida — «casi instantánea»-, debido a revoluciones genéticas, las cuales interpretaban de una manera parecida a la de Richard Goldschmidt, en el sentido de mutaciones puntuales que tendrían grandes efectos fenotípicos que conducirían al inmediato surgimiento de taxones de alto rango.

Bajo esta nueva visión, la especiación resultaba ser un fenómeno evolutivo causado por un proceso macroevolutivo propio, irreductible a los procesos de cambio microevolutivo que operan dentro del marco del medio ambiente y la constitución biológica de una especie. En esta versión radicalizada de la teoría se presenta de manera más enfática la tesis original de que el cambio fenotípico gradual está prácticamente ausente en la evolución de los linajes filéticos, y que los períodos de completa estasis fenotípica de las especies son interrumpidos únicamente mediante eventos de especiación. Adicionalmente, se disminuye la importancia de la adaptación, con la consiguiente disminución del papel de la selección natural, y se juega con la idea de que las macromutaciones (probablemente debidas a nuevos arreglos cromosómicos) podrían provocar cambios importantes en las especies en el lapso de sólo una o dos generaciones. Curiosamente, el acento se traslada de Charles Darwin a Richard Goldschmidt.

La teoría del equilibrio puntuado se presentaba entonces como una alternativa a la síntesis moderna y como tal la abrazaron sus partidarios o la criticaron sus detractores.

20 Es en este artículo donde se declara «muerta» a la síntesis moderna: «I have been reluctant to admit it, but if Mayr's characterization of the synthetic theory is accurate, then that theory as a general proposition is effectively dead». GOULD, S. J. (1980), «Is a New and General Theory of Evolution Emerging?», Paleobiology, 6, pp. 119-130, 120.

21 Hoffman, A. (1992), «Twenty Years Later: Punctuated Equilibrium in Retrospect», en SOMIT, A.; Peterson, S. (ed.), The Dynamics of Evolution. The Punctuated Equilibrium Debate in the Natural and Social Sciences, Ithaca, Cornell University Press, pp. 121-138.

22 RuSE, M. (1992), «Is the Theory of Punctuated Equilibria a New Paradigm?», en SomIT, A.; PETERSON, S. (ed.), The Dynamics of Evolution. The Punctuated Equilibrium Debate in the Natural and Social Sciences, Ithaca, Cornell University Press, pp. 139-167.

23 Gould, S.J.; Eldredge, N. (1977); Gould, S.J. (1980); Gould, S.J. (1982b), «Darwinism and the Expansion of Evolutionary Theory», Science, 216, pp. 380-387; STANLEY (1979), (1982a), (1982b). 
Siguiendo la terminología de Lakatos $^{24}$, podemos decir que se la presentaba como un programa de investigación que superaría a su rival gracias a que era capaz de acumular más contenido empírico a su favor, concretamente el contenido del registro fósil (lo que, como veremos en la cuarta parte de este trabajo, no resultó tan exacto).

Pero, y a pesar de sus pretenciones manifiestas, la teoría no consiguió sustituir a la síntesis moderna. O, al menos, no lo ha logrado hasta ahora. La paradoja estriba, entonces, en que a pesar de esto el equilibrio puntuado haya sobrevivido. El hecho constatable de su supervivencia será mostrado en la siguiente sección, y sus causas probables las exploramos en la tercera parte de este trabajo.

\section{II. ¿HA SOBREVIVIDO LA TEORÍA DEL EQUILIBRIO PUNTUADO?}

\section{CUADRO 1}

ARTÍCULOS APARECIDOS EN PALEOBIOLOGY Y EN EVOLUTION, FIRMADOS POR AUTORES DISTINTOS A GOULD Y ELDREDGE, QUE CITAN OBRAS DEL EQUILIBRIO PUNTUADO

\begin{tabular}{|c|c|c|c|}
\hline $\begin{array}{c}\text { Años de las } \\
\text { publicaciones }\end{array}$ & $\begin{array}{c}\text { Artículos en } \\
\text { Paleobiology que citan } \\
\text { al equilibrio puntuado }\end{array}$ & $\begin{array}{c}\text { Articulos en Evolution } \\
\text { que citan al equilibrio } \\
\text { puntuado }\end{array}$ & $\begin{array}{c}\text { Total de artículos } \\
\text { con citas al respecto }\end{array}$ \\
\hline $1975-79$ & 18 & 6 & 24 \\
\hline $1980-84$ & 42 & 22 & 64 \\
\hline $1985-89$ & 30 & 7 & 37 \\
\hline $1990-94$ & 9 & 15 & 24 \\
\hline $1995-00$ & 27 & 13 & 40 \\
\hline Total: & $\mathbf{1 2 6}$ & $\mathbf{6 3}$ & $\mathbf{1 8 9}$ \\
\hline
\end{tabular}

Nota: Este cuadro se construyó a partir del cuadro elaborado por Ruse ${ }^{25}$ para el período 19751995; los datos para el período 1995-2000 fueron completados por nosotros.

Para poder responder a esta pregunta analizaremos cuántos artículos han hecho referencia al equilibrio puntuado, entre todos aquellos artículos aparecidos en las dos revistas científicas más relevantes con relación al contenido de esta teoría: Paleobio$\log y^{26}$ y Evolution. Consideramos que si el equilibrio puntuado ha tenido algún grado

24 LaKatos, I. (1982), La metodología de los programas de investigación cientifica, Madrid, Alianza Editorial.

25 RUSE (1999).

26 Gould y Eldredge, como paleontólogos que son, han participado entusiastamente en la revista especializada en paleontología y paleobiología Paleobiology desde su fundación, en 1975. Gould, incluso, es 
de éxito dentro de la comunidad científica es en estas publicaciones especializadas, más que en ninguna otra, donde esta aceptación debería evidenciarse. Al hacer esto, estamos aceptando el criterio de Ruse $^{27}$ de que una teoría sigue viva en tanto sea citada y en tanto continúen las referencias a la misma en trabajos posteriores.

Como puede apreciarse, el promedio de artículos con citas a la teoría, visto de manera conjunta, ha sido de 38 cada cinco años. El mayor interés se alcanzó, en ambos casos, entre 1980 y 1984 (durante la etapa radical de la teoría), y después de una aparente declinación las cifras han retornado a su promedio histórico.

Analicemos ahora, con mayor detalle, lo ocurrido durante el último período que presentamos (1995-2000) en cada una de las revistas. En el siguiene cuadro sobre Paleobiology se puede apreciar tanto la frecuencia de publicación como el número de artículos que emplearon citas sobre obras de la teoría del equilibrio puntuado en cada uno de estos años:

\section{CUADRO 2}

Números de PALEOBIOLOGY CON ARTículos DE AUTORES Distintos A GOULD Y ELDREDGE QUE CITAN OBRAS DEL EQUILIBRIO PUNTUADO

\begin{tabular}{|c|c|c|c|c|c|}
\hline $\begin{array}{c}\text { Número de la } \\
\text { revista y año de } \\
\text { publicación }\end{array}$ & $\# 1$ & $\# 2$ & $\# 3$ & $\# 4$ & $\begin{array}{c}\text { Total de } \\
\text { artículos con } \\
\text { citas }\end{array}$ \\
\hline 1995 & 2 & 2 & & & 4 \\
\hline 1996 & 2 & 2 & 3 & & 7 \\
\hline 1997 & & 1 & 1 & & 2 \\
\hline 1998 & 3 & 2 & 2 & 1 & 8 \\
\hline 1999 & 1 & & 1 & 1 & 3 \\
\hline 2000 & & & 2 & 1 & 3 \\
\hline Total: & & & & & $\mathbf{2 7}$ \\
\hline
\end{tabular}

Durante 1995-2000 esta revista publicó 200 artículos. Entre estos, hubo 27 artículos (de autores distintos a Gould y Eldredge) que emplearon en alguna medida la teoría en su texto, puesto que la citan (en 51 ocasiones), a lo largo de los 6 años y los

mencionado en las contraportadas como uno de los patrocinadores económicos de la revista. Desde su aparición inicial, esta revista se ha ganado un lugar destacado dentro del campo de las publicaciones profesionales en paleontología e historia natural. Por todas estas razones, es comprensible que la publicación científica natural para publicar trabajos sobre el equilibrio puntuado haya sido, precisamente, Paleobiology.

27 Ruse, M. (1999), Mystery of Mysteries. Is Evolution a Social Construction?, Massachusetts, Harvard University Press, pp. 135-152. 
24 números de la revista que se analizaron. Esta cifra significa que el 13,5\% (27/200) de los artículos publicados en este lapso han hecho referencia a la teoría. Además, y como puede apreciarse, en la mayor parte de las revistas ha aparecido al menos un artículo con citas al respecto: el porcentaje de números (revistas) de Paleobiology que incluyeron citas sobre el tema, en comparación con el total de números publicados en este período, es del 67\% (16/24).

Y sin embargo, cuando analizamos con mayor detalle estos seis años, podemos notar que la mayoría de las citas que han aparecido corresponden a meras referencias secundarias, a citas de pasada, y que los artículos que las incluyen no participan de la teoría ni de la polémica que inicialmente despertó. ¿Qué artículos sostuvieron, realmente, a la teoría del equilibrio puntuado como un modelo alternativo de evolución? Apenas los siguientes cuatro entre 1995 y el 2000:

- Lieberman, B.S.; Brett, C.; Eldredge, N. (1995), «A study of stasis and change in two species lineages from the Middle Devonian of New York State», Paleobiology, 21, pp. 15-27.

- Norris, R.D.; Corfield, R.M.;Cartlidge, J. (1996), «What is gradualism? Cryptic speciation in globarotaliid foraminifera», Paleobiology, 22, pp. 386-405.

- Miller, A.I. (1997), «Coordinated stasis or coincident relative stability?», $P a-$ leobiology, 23, pp. 155-164.

- Chiba, S. 1998. «Synchronized evolution in lineages of land snails in oceanic islands». Paleobiology 24, pp. 99-108.

Hagamos a continuación el mismo ejercicio para el caso de Evolution, la cual edita seis números al año:

\section{CUADRO 3}

NÚMEROS DE EVOLUTION CON ARTí́CULOS DE AUTORES DISTINTOS A GOULD Y ELDREDGE QUE CITAN OBRAS DEL EQUILIBRIO PUNTUADO

\begin{tabular}{|c|c|c|c|c|c|c|c|}
\hline $\begin{array}{c}\text { Número de la } \\
\text { revista y año de } \\
\text { publicación }\end{array}$ & $\# 1$ & $\# 2$ & $\# 3$ & $\# 4$ & $\# 5$ & $\# 6$ & $\begin{array}{c}\text { Total de artículos } \\
\text { con citas }\end{array}$ \\
\hline 1995 & & 2 & & & 1 & & 3 \\
\hline 1996 & & 1 & & 2 & & 1 & 4 \\
\hline 1997 & & & 2 & & 1 & & 3 \\
\hline 1998 & & & 1 & 1 & & & 2 \\
\hline 1999 & & & & & & & 0 \\
\hline 2000 & & 1 & & & & & 1 \\
\hline Total: & & & & & & & $\mathbf{1 3}$ \\
\hline
\end{tabular}


Durante 1995-2000 Evolution publicó 1.065 artículos. Ahora bien, hay que tomar en cuenta que el contenido temático de esta revista es mucho más amplio que el de Paleobiology, abarcando no sólo asuntos de macroevolución sino temas tan diversos como la evolución a nivel molecular, estudios de campo y experimentos de laboratorio, evolución en plantas y estudios de genética de poblaciones. Por consiguiente, era de esperarse una menor representación de artículos con interés en la teoría del equilibrio puntuado en relación a lo encontrado en Paleobiology. A pesar de esto, y como puede apreciarse, hubo 13 artículos (de autores distintos a Gould y Eldredge) que emplearon en alguna medida la teoría en su texto, y que la citan (en 19 ocasiones), a lo largo de los 6 años y los 36 números analizados de esta revista. Esta cifra significa que el 1,2\% (13/1.065) de los artículos publicados en este lapso han hecho referencia al modelo del equilibrio puntuado. Además, la presencia del puntuacionismo también puede medirse en el hecho de que en casi todos estos años - la excepción es el año de 1999- han aparecido entre uno y cuatro artículos que citan la teoría. El porcentaje de números (revistas) de Evolution que incluyeron citas sobre el tema, en comparación con el total de números publicados en este período, es del $28 \%(10 / 36)$; es decir, uno de cada tres números, en promedio, ha publicado algún artículo que sigue citando en su bibliografía obras relativas a la teoría del equilibrio puntuado.

Pero, nuevamente, cabe preguntarse ¿cuántos de estos artículos trataron, realmente, sobre el tema del equilibrio puntuado como patrón alternativo de evolución? Esta vez, tan sólo dos entre 1995 y el 2000:

- Marko, P. B. (1998), «Historical allopatry and the biogeography of speciation in the Prosobranch Snail genus Nucella.», Evolution, 52, pp. 757-774.

- Samadi, S.; David, P.; Jarne, P. (2000), «Variation of shell shape in the clonal snail Melanoides tuberculata and its consequences for the interpretation of fossil series», Evolution, 54, pp. 492-502.

A partir de estos datos podemos concluir que: 1) La teoría del equilibrio puntuado ha permanecido viva, desde su surgimiento y hasta ahora, a través de referencias en las páginas de - por lo menos - estas dos revistas especializadas; 2) sin embargo, el interés por la misma ha declinado, ya que la gran mayoría de las citas no se refieren a artículos que traten directamente sobre el tema, ya sea respaldando o rechazando el modelo puntuado. En otras palabras, el debate entre el equilibrio puntuado y la síntesis moderna ha perdido intensidad y también presencia en ambas publicaciones. 


\section{III. ¿POR QUÉ EL EQUILIBRIO PUNTUADO DERIVÓ EN UNA TEORÍA DE RANGO MEDIO?}

Para Imre Lakatos ${ }^{28}$ la unidad descriptiva típica del quehacer científico no es una teoría aislada, ni un conjunto de teorías, sino un programa de investigación. Cada programa de investigación cuenta con un núcleo firme, «tenazmente protegido contra las refutaciones mediante un gran cinturón protector de hipótesis auxiliares. Y, lo que es más importante, el programa de investigación tiene también una heurística, esto es, una poderosa maquinaria para la solución de problemas que... asimila las anomalías e incluso las convierte en evidencia positiva ${ }^{29}$. Éste es el caso de la síntesis moderna; no se trata de una teoría aislada, inmóvil desde la década de los cuarenta del siglo pasado, sino de un verdadero programa de investigación, en torno al cual hay una activa comunidad científica que continúa investigando y aportando nuevos conocimientos.

Lakatos sostiene que los programas de investigación también pueden ser caracterizados por medio de su heurística, tanto positiva como negativa. «La heurística negativa especifica el núcleo firme del programa, que es irrefutable por decisión metodológica de sus defensores; la heurística positiva consiste de un conjunto, parcialmente estructurado, de sugerencias o pistas sobre cómo cambiar y desarrollar las versiones refutables del programa de investigación, sobre cómo modificar y complicar el cinturón protector refutable.» ${ }^{30}$

Según este esquema, «...las anomalías no se aceptan como refutaciones del núcleo firme sino como refutaciones de algunas hipótesis del cinturón protector. En parte, debido a la presión empírica (pero, en parte, según el diseño de su heurística) el cinturón protector es modificado constantemente, expandido, complicado, mientras que el núcleo firme permanece intacto» ${ }^{31}$. Con el objeto de que el cinturón protector sea el que reciba los impactos de las refutaciones, podrá ser ajustado continuamente e incluso completamente sustituido.

En este momento de nuestra exposición necesitamos aclarar, entonces, qué entendemos por el núcleo firme de la síntesis moderna (y, por exclusión, a todas las demás hipótesis de la teoría sintética de la evolución las consideraremos, de momento, como parte de su cinturón protector). Desde nuestro punto de vista, el núcleo firme de la síntesis moderna está constituido por: a) la aceptación de la selección natural como el principal mecanismo evolutivo y el único que produce adaptación; b) un enfoque poblacional para los cambios evolutivos; c) la aceptación de que cualquier mecanismo evolutivo debe ser consistente con lo que sabemos de genética molecular.

\footnotetext{
28 LAKATOS (1982).

29 Ibidem, p. 13.

30 Ibidem, p. 68

31 Ibidem, p. 230.
} 
Ahora bien, de acuerdo con Robert Merton ${ }^{32}$ se debe considerar como teorías de rango medio aquellas teorías a medio camino entre las hipótesis de trabajo diario que se emplean en la investigación y las teorías generales. Las teorías de rango medio tienen que ver con aspectos delimitados de los fenómenos. Por ello, tratan casos particulares con mayor detalle que las teorías generales en donde el nivel de abstracción suele alejarlas del detalle.

El término, sin embargo, no pretende ser peyorativo en modo alguno. Para Schaffner ${ }^{33}$ estas teorías son, de hecho, las más frecuentes tanto en la biología como en la medicina y son importantes ya que sirven para organizar sus áreas de estudio además de que, normalmente, son teorías que ofrecen parámetros medibles. Por su parte, Merton ${ }^{34}$ emplea el ejemplo de la física como una ciencia que ha crecido y está caracterizada por este tipo de teorías de rango medio que, con el tiempo, se han ido agrupando en familias de teorías.

Una vez aceptado su papel en el desarrollo de la ciencia, debemos insisitir, no obstante, en que una teoría de rango medio sólo es aplicable a un rango conceptual limitado. Puede ser que, con el tiempo, evolucione progresivamente hacia niveles de generalización superiores y que llegue a converger con otras teorías de rango medio para consolidar grupos especiales de teorías, como en el caso de la física. Pero no está en su diseño el convertirse directamente en una teoría de aplicación general. No son - y no lo serán en el futuro previsible - teorías «universales». Sin embargo, sí son teorías que han logrado estabilizarse (dentro de su rango de aplicación) y, por lo tanto, sobrevivir.

Éste es el sentido en que aplicamos el término en este artículo. En el resto de nuestra exposición procuraremos mostrar que, a pesar de que la teoría del equilibrio puntuado fue presentada como una nueva teoría general de la evolución, no sólo no consiguió serlo sino que ha derivado en una teoría de aplicación limitada, con las ventajas de ofrecer algunos casos muy particulares en los que muestra evidencia empírica medible a su favor, pero cuyo contenido, sin embargo, no le permite derivar las conclusiones de alcance general que desearía.

Veamos ahora qué circunstancias y qué razones causaron que el equilibrio puntuado derivara en la actualidad hacia una teoría de rango medio. Hemos identificado, al menos, tres factores diferentes:

\footnotetext{
32 Merton, R. (1968), «On Sociological Theories of the Middle-Range», en Social Theory and Social Structure, Nueva York, Free Press, pp. 39-53.

33 SChaffner, K. F. (1993), Discovery and Explanation in Biology and Medicine, Chicago, The University of Chicago Press, p. 98.

34 MERTON (1968).
} 
PRIMER FACTOR: retrocedió el radicalismo de los autores puntuacionistas

En primer lugar, hizo falta enfriar los ánimos y permitir que la retórica — que caracterizó la polémica en torno al equilibrio puntuado durante su primer década de existencia - diera pie a un análisis más serio sobre las propuestas centrales de la teoría. Esto sólo fue posible, sin embargo, cuando el radicalismo del puntuacionismo retrocedió para dejar en su lugar una versión menos polémica de la teoría.

Como es natural suponer, la radicalización del equilibrio puntuado en su segunda etapa provocó una reacción frontal de parte de los autores de la síntesis moderna, y es durante estos años que la polémica entre los exponentes de ambas teorías alcanzó su punto más álgido.

Pero, hasta los teóricos de la evolución invocados por Gould y Eldredge para apoyar las implicaciones del equilibrio puntuado, comenzaron a criticar la idea de la especiación como un proceso macroevolutivo debido a revoluciones genéticas ${ }^{35}$, ya que incluso si tales revoluciones tuvieran lugar, la aparición de una nueva especie de todos modos tendría que darse gradualmente, ya que el cambio siempre es un proceso poblacional.

A los puntuacionistas que sostenían la versión radical se les reprochó, también, que el mero concepto de una macromutación como el principal responsable de un proceso de especiación era muy poco plausible. Pues, a pesar de que no hay una razón lógica para descartar que un mecanismo así pueda operar en la naturaleza, toda la evidencia genética apunta a que las mutaciones puntuales que tienen un gran efecto en el fenotipo generalmente son adaptativamente desventajosas, e, incluso, cuando no lo son, no conllevan a la especiación.

Por todo esto, la versión radical del equilibrio puntuado y sus implicaciones macroevolutivas resultaron difícilmente defendibles y de hecho, el propio Gould ${ }^{36}$, ante el alud de críticas, dio marcha atrás en su idea de la formación instantánea de nuevas especies.

A partir de la segunda mitad de los ochenta no volvieron a publicarse artículos del equilibrio puntuado con referencias a Goldschmidt y, de hecho, desde entonces se ha buscado desligar a la teoría de cualquier connotación saltacionista. Comienza así lo que Ruse ${ }^{37}$ ha denominado la tercera fase de la teoría, a partir de la cual se modula la idea saltacionista de cambios macroevolutivos instantáneos, y lo que se argumenta ahora es que 50.000 años, si bien pueden ser muchos para los genetistas que estudian la mosca de la fruta, son apenas «un instante» para los paleontólogos.

35 MAYR, E. (1982), «Process of speciation in animals», en BARIGOZZI, C. (ed.) Mechanisms of Speciation, Nueva York, Alan R. Liss, pp. 1-20; WRIGHT, S. (1982), «The shifting balance theory and macroevolution», Annu. Rev. Gen., 16, pp. 1-19.

36 GOULD, S. J. (1985), «The paradox of the first tier: an agenda for paleobiology», Paleobiology, 11, pp. 2-12.

37 RUSE (1992). 
El primer paso, que fue un paso hacia atrás en las posiciones extremas que se habían asumido, estaba dado.

\section{SEGUNDO FACTOR: el núcleo firme de la síntesis moderna no fue refutado}

La segunda razón del estatus de rango medio que actualmente tiene el puntuacionismo es que la mayoría de sus propuestas - contra lo que la retórica de la controversia inducía a creer - no atentaban, en realidad, contra el núcleo firme de la síntesis moderna, y sólo implicaron modificaciones en su cinturón protector.

Una vez abandonada la versión saltacionista — o cuasi saltacionista - fue posible centrar el debate en los puntos relevantes de la teoría del equilibrio puntuado. Pero, ¿cuáles eran las propuestas relevantes? En primer lugar, estaba la idea de que las especies transcurren la mayor parte de su vida evolutiva en estasis, contra el cambio gradual que cabría esperar en el supuesto de anagénesis. En segundo lugar, la teoría predecía que la mayor parte de los cambios ocurrirían durante los eventos de especiación, además de que dichos eventos de especiación normalmente ocurren bajo el esquema - propuesto originalmente por Mayr - del aislamiento geográfico de poblaciones (situadas en la periferia del rango principal de distribución de la especie). Por último, se estaba proponiendo una visión jerárquica de los procesos evolutivos: en el nivel del gen podían ocurrir fenómenos no necesariamente adaptativos - como la deriva génica-; al nivel del organismo actuaría la selección natural (aunque limitada por las constricciones genéticas del organismo); por último, al nivel de las especies operarían dinámicas evolutivas propias — como la selección al nivel de especies-, imposibles de reducir a los niveles jerárquicos inferiores.

Pero, ¿cuáles de estas propuestas atentaban, realmente, contra al núcleo teórico de la síntesis moderna, contra su núcleo firme? ¿Era posible absorber estas propuestas y refutaciones en el cinturón protector?

La idea central del puntuacionismo, la de que la historia evolutiva de las especies consiste principalmente de largos periodos de estasis fenotípica - o, cuando menos, de estasis morfológica - no encerraba, en su versión original, una amenaza significativa para el núcleo firme de la síntesis moderna. Después de todo, desde antes de que la teoría del equilibrio puntuado llamara la atención sobre el fenómeno de la estasis, se conocía ya una variedad de mecanismos biológicos - concordantes con la síntesis moderna - que podrían explicar la estasis fenotípica como, por ejemplo, la selección estabilizadora o la coevolución homeostática ${ }^{38}$. La estasis, en este sentido, era una refutación ya asimilada por el cinturón protector de hipótesis auxiliares.

\footnotetext{
38 HOFFMAN (1992).
} 
Sin embargo, cuando el equilibrio puntuado entró en su segunda etapa, la versión de la estasis también se radicalizó: ahora se sostenía que el registro fósil presentaba varios casos de estasis fenotípica incluso a lo largo de períodos en los que se habían experimentado cambios importantes en el medio ambiente y, por tanto, en las presiones de selección. ¿Qué hacer con este concepto de estasis que resiste a los cambios en las presiones de selección? Esta nueva definición sí representó una refutación que debía atenderse con más seriedad y que, de comprobarse, no sería fácilmente absorbida por el cinturón protector. Sin embargo, primero debería ser explicada por los propios autores puntuacionistas: ¿qué proponían ellos mismos para explicar el supuesto fenómeno? Eldredge ${ }^{39}$ ha argumentado que, cuando el medio ambiente cambia, la respuesta más probable de los organismos no es adaptarse a las nuevas condiciones, sino trasladarse a un hábitat similar a aquel para el que ya se encontraban adaptados. De este modo, las especies permanecerían sin cambio incluso cuando su medio ambiente cambiaba. Pero esta explicación de Eldredge únicamente aplica a los casos en que sólo una parte del medio ambiente cambia y en que las poblaciones son capaces de buscar y encontrar un hábitat equivalente al que han perdido. $\mathrm{Y}$, en los casos en que puede proceder, el argumento de Eldredge no refuta las tesis centrales de la síntesis moderna.

La heurística de la teoría sintética de la evolución agregó, además, otro argumento en su propia defensa: no hay manera de probar que la estasis morfológica mostrada por el registro fósil corresponda a una total estasis fenotípica y, tampoco, de probar que no hayan existido variaciones morfológicas en racimo que, al no ser lo suficientemente comunes, no quedaron preservadas ${ }^{40}$.

Otra idea del puntuacionismo, la relativa a la especiación peripátrica, no necesitó siquiera ser desviada al cinturón protector, ya era parte constitutiva de ese mismo cinturón y la propuso precisamente uno de los fundadores de la síntesis moderna ${ }^{41}$. Antes del lanzamiento formal de la teoría, el mismo Eldredge ${ }^{42}$ estaba de acuerdo en que aplicar el mecanismo de Mayr a la paleontología implicaba más una ordenación de la paleontología con la nueva síntesis que un enfrentamiento con ésta. Sin embargo, en el artículo conjunto con Gould ${ }^{43}$ éste último lo convenció de darle un giro al enfoque, y entonces se añadió una idea provocativa: que los cambios evolutivos ocurrían casi únicamente durante estos eventos de especiación.

\footnotetext{
39 ELDREDGE (1995).

40 Este concepto, que será ampliado más adelante, fue propuesto por WiLLIAMS, G. (1992), Natural Selection, Domains, Levels, and Challenges, Nueva York, Oxford University Press, pp. 127-142.

41 MAYR (1954).

42 ELDREDGE (1971).

43 ELDREDGe,N.; GOULD, S.J. (1972)
} 
Aunque esta propuesta se presentaba como consistente con la síntesis moderna, desde el lado rival no fue bien recibida. Nuevamente fue desviada hacia el cinturón protector, no había evidencia suficiente que la soportara y, por el contrario, sí abundaban ejemplos reconocidos de linajes que habían evolucionado por anagénesis a lo largo de períodos en los que no se halla evidencia de eventos de cladogénesis. Incluso el mismo Mayr ${ }^{44}$ se declaró sorprendido por la manera tan categórica en que había sido empleada su propuesta dentro de las tesis del puntuacionismo, rechazando que su mecanismo de especiación peripátrica mediante revoluciones genéticas implicara que los cambios evolutivos deberían concentrarse durante los eventos de especiación.

Finalmente, queda la propuesta puntuacionista de los niveles jerárquicos de selección. Pero, y a pesar de las múltiples publicaciones en que se hace mención de ella, esta idea aún no está del todo definida.

Según esta propuesta teórica, en el momento en que una especie va a ser reemplazada por otra en la línea evolutiva, varias especies hijas nuevas son candidatas a la sucesión, cada una de ellas provista de diferentes adaptaciones particulares a ambientes distintos (adaptaciones que no están orientadas en relación con alguna determinada tendencia evolutiva, sino distribuidas al azar). Pero, si un ambiente dado resulta más favorable a la expansión de una especie particular dotada de la correspondiente adaptación, esa especie persistirá duraderamente, en detrimento de las otras, que se extinguirán. Así pues, ocurre un proceso de selección entre especies, al cual se lo presenta como independiente de la selección natural que se pueda dar al interior de las poblaciones de cada especie. Para el puntuacionismo, no se trata de una expresión a otro nivel de un solo fenómeno, sino de la comprobación de la existencia de diversos fenómenos de selección, distintos al de la mera selección individual, y que aparecen como propiedades emergentes a diferentes niveles jerárquicos ${ }^{45}$.

Sin embargo, para la síntesis moderna esto no ha representado un problema teórico. Después de todo, lo más que podría hacer la selección de especies sería elegir entre varias alternativas de organismos con un nivel dado de complejidad, pero este nivel de complejidad habría sido logrado previamente, y por entero, mediante selección natura ${ }^{46}$. Además, y puesto que la selección de especies se efectuaría mediante el mecanismo de selección individual de los organismos que conforman las poblaciones de

\footnotetext{
44 MAYR (1992).

45 Estas ideas fueron desarrolladas en ELDREDGE, N. (1982), «Phenomenological levels and evolutionary rates», Syst. Zool., 31, pp. 338-347; (1985), Unfinished Synthesis: Biological Hierarchies and Modern Evolutionary Thought, Nueva York, Oxford University Press, pp. 128-135 y 160-174; (1988), Macroevolutionary Dynamics, Nueva York, MacGraw-Hill; (1995); (1999); y también en GoULD, S. J. (1982a), «The meaning of punctuated equilibrium and its role in validating a hierarchical approach to macroevolution», en MilkMAn, R. (ed.) Perspectives in Evolution, Massachusetts, Sinauer, pp. 83-104; (1982b); (1985); (2002).

46 DAWKINS (1996)
} 
las especies que son llevadas a la extinción, en realidad estaríamos hablando simplemente del fenómeno de sucesión de especies ${ }^{47}$, pero no de un fenómeno nuevo.

Así pues, el concepto de selección al nivel de especies, si bien se lo quiere presentar como un fenómeno distinto al de selección natural al nivel del individuo (aunque equivalente en el nivel de especies), no ha ido acompañado, hasta ahora, de un mecanismo claro que permita comprender si de verdad se trata de un fenómeno distinto, o sólo una expresión a otro nivel de un solo mecanismo: el de la selección natural al nivel del individuo. Un inconveniente más serio es que, hasta ahora, esta propuesta no cuenta con una base genética. Por lo tanto, no puede ser una amenaza para el núcleo firme de la síntesis moderna.

En los tres casos que hemos descrito, los intentos de refutación al núcleo firme de la teoría sintética de la evolución han sido desestimados por falta de evidencia empírica suficiente, o bien han sido tomados como meras precisiones a algunas ideas auxiliares, pero no como amenazas a sus tesis esenciales.

TERCER FACTOR: la incorporación de algunos de los conceptos clave del equilibrio puntuado en el cinturón protector de la síntesis moderna

Un tercer factor, muy ligado al anterior, lo constituye el hecho de que la síntesis moderna ha logrado asimilar algunos conceptos clave del puntuacionismo (como la estasis y la especiación rápida) dentro del cuerpo teórico de su propio cinturón protector de hipótesis auxiliares, sin mayores contratiempos. De esta manera, algunos de los conceptos del puntuacionismo han sobrevivido dentro de la teoría que pretendían criticar, volviéndose así, de cierta manera, inofensivos.

Veinte años después de que Eldredge y Gould presentaran el concepto de la estasis como uno de los más desafiantes al cuerpo teórico de la síntesis moderna, nos encontramos con que los autores más ortodoxamente darwinistas no sólo no lo estaban rechazando, sino que ya lo habían incorporado a sus obras; el concepto fue lo suficientemente importante como para que Williams ${ }^{48}$ le dedicara todo un capítulo en Natural Selection, Domains, Levels and Challenges. Asimismo, Mayr ${ }^{49}$ ocupa toda una seccion a hablar de la estasis morfológica y sus posibles causas. Hasta cierto punto, podría decirse que la teoría sintética de la evolución se apropió de la estasis, la convirtió en parte de su programa de investigación, y con ello le quitó toda connotación antidarwinista.

47 MAYR, E. (1997), This es Biology. The Science of the Living World, Cambridge, Harvard University Press, pp. 195-203.

48 WILLIAMS (1992).

49 MAYR (1992). 
Sin embargo, la estasis para los exponentes de la síntesis moderna no necesariamente significa lo mismo que para Gould y Eldredge. Mientras que para los puntuacionistas implica una fuerte estabilidad fenotípica, debida principalmente a una probable cohesión del genotipo que prevalece sobre eventuales presiones de selección, para Williams ${ }^{50}$ es un suceso más bien estadístico que en nada implica algo parecido a una cohesión genotípica. Williams propone, para explicar lo que él llama estasis taxonómica, que lo que puede aparecer en el registro fósil como una estasis completa esconde, en realidad, una continua variabilidad que no aparece registrada por el hecho de haberse dado más bien en forma de racimo que en forma de árbol: es decir, las variaciones morfológicas pueden haberse presentado constantemente, pero sin alejarse nunca demasiado de la forma ancestral, la cual resulta mucho más constante a lo largo del tiempo geológico por haber estado sujeta a una selección normalizadora de clado. Así, cada nueva variante tendría períodos de vida geológicamente tan cortos que sus representates no quedarían conservados en el registro fósil, y lo que veríamos serían sólo los fósiles de la forma ancestral, favorecida por la selección de clado. Esto produciría una apariencia de estasis completa e, inclusive, de eventos de evolución puntuada.

Así pues, Williams acepta la estasis, pero con otro significado: no se trata de una ausencia de cambios macroevolutivos, sino de la tendencia a que, dentro de un gran número de variedades, prevalezca la forma ancestral debido a la presión normalizadora de la selección de clado. Se trata de la prevalencia de la forma ancestral a pesar - y no en ausencia, como sería para los puntuacionistas - de las variaciones morfológicas producidas.

Mayr ${ }^{51}$ también habla de la estasis como un fenómeno compatible con la síntesis moderna. Pero, a diferencia de Williams, él no tiene problema en aceptar que su causa probable sea una cohesión del genotipo - después de todo, el propio $\mathrm{Mayr}^{52}$, junto con Chetverikov ${ }^{53}$ y Lerner ${ }^{54}$, es uno de los padres de la idea-, y también discrepa con Williams acerca de que la paleontología no sea el camino indicado para dilucidar el asunto. Por el contrario, sostiene que una cuestión como la estasis no puede ser resuelta ni con la teoría genética ni mediante el estudio de especies vivas, y que solamente podrá resolverse a través del análisis de evidencias paleontológicas. En este sentido, su concepción de la estasis es la que más se acerca, dentro de los exponentes de la síntesis moderna, a la concepción de los propios autores puntuacionistas.

50 WiLLIAMS (1992).

51 MAYR (1992).

52 MAYR (1954).

53 Chetverikov, S. (1926), «On certain aspects of the evolutionary process from the standpoint of modern genetics», J. Exp. Biol., A2, pp 3-54.

54 LERNER, I.M. (1954), Genetic Homeostasis, Nueva York, Wiley. 
Pero la aceptación del fenómeno de la estasis por parte de Mayr no implica una aceptación del equibrio puntuado y, ni siquiera, de que la estasis predomine sobre la evolución gradual. En todo caso la ve como un fenómeno alternativo y sostiene que lo mejor que podría hacer uno es adoptar una posición intermedia, aceptando que en muchas especies se presenta una evolución gradual y, al mismo tiempo, admitiendo que hay un número inesperadamente alto de especies fósiles que, en efecto, no muestran cambios morfológicos a lo largo de varios millones de años.

Dawkins ${ }^{55}$, por su parte, también dedica un capítulo completo a hablar de la perfecta compatibilidad de la síntesis moderna con los eventos de rápida especiación que, además, pueden contrapuntearse con períodos de estasis. Tampoco la evolución rápida, y por ende los eventos de especiación rápida, fueron tomados como algo que no estuviera ya contemplado en el cinturón protector de ideas auxiliares del programa de investigación de la teoría sintética de la evolución. Por más que Gould ${ }^{56}$ insista en que el gradualismo darwinista descarta los eventos de rápida evolución, existe un consenso entre los representantes de la síntesis moderna respecto a que el gradualismo no implica, en modo alguno, un tasa lenta y constante de cambio fenotípico, sino tan sólo que los cambios, cuando ocurren, son necesariamente graduales en el sentido de que únicamente a través de sucesivas generaciones pueden extenderse dentro de una población, condición previa para dar origen a una nueva especie.

En conclusión, tanto el fenómeno de la estasis como el de la evolución rápida es decir, de la evolución con tasas variables e intermitentes de cambio fenotípicohan dejado de ser las armas más poderosa de la teoría del equilibrio puntuado en contra del núcleo firme de la teoría sintética de la evolución. En la actualidad ambos conceptos han sido incorporados al cinturón de ideas auxiliares que protege a la síntesis moderna, si bien suavizados y, en ocasiones, con significados e implicaciones algo diferentes entre un autor y otro.

\section{LAS DIFICULTADES EMPÍRICAS PARA PODER VALIDAR O REFUTAR LA TEORÍA DEL EQUILIBRIO PUNTUADO MEDIANTE EL REGISTRO FÓSIL}

Además de los tres factores ya descritos, nosotros creemos que hay dificultades empíricas reales que también han jugado un papel importante en la suerte que ha corrido el equilibrio puntuado. Desde su surgimiento ha sido difícil «poner a prueba» esta teoría. La naturaleza del registro fósil ha hecho que resulte sumamente difícil si no es que imposible - obtener evidencia irrefutable tanto para los casos de gradua-

\footnotetext{
55 DAWKINS (1996).

56 Gould (1980); (1982b).
} 
lismo como para los de estasis (o para los casos de especiación ocurridos durante períodos muy breves de tiempo).

En opinión de algunos investigadores ${ }^{57}$ los resultados paleontológicos nunca serán decisivos, pues el registro fósil no permite identificar inequívocamente si tenemos un patrón gradual o puntuado a lo largo de la evolución de una especie. Y es que, para poder demostrar más allá de toda duda que ocurrió una evolución gradual, no basta con que uno pruebe con el registro fósil que un grupo orgánico sufrió un cambio fenotípico gradual y significativo a lo largo del tiempo en cierta área geográfica. Uno tendría que mostrar, también, que dicha área era lo suficientemente grande y ecológicamente heterogénea como para poder descartar la explicación alternativa de que el aparente cambio fenotípico gradual obedece, en realidad, a eventos de inmigración de otra población de la misma especie, o incluso de una especie cercanamente emparentada. Y esta tarea es muy difícil —e incluso, a veces, imposible - de llevar a cabo, ya que requiere de correlaciones precisas del tiempo en que ocurrieron diversos eventos biológicos, que además tuvieron lugar en una variedad de áreas, a veces muy distantes una de las otras. Sin embargo, y a pesar de todas estas dificultades, la literatura paleontológica contiene un número considerable de ejemplos convincentes de cambios fenotípicos graduales.

Pero si el cambio fenotípico gradual es difícil de probar más allá de toda duda, resulta todavía más complicado demostrar o refutar casos de evolución puntuada mediante el registro fósil. Aparte de los muy raros casos que se han documentado de evidencia paleontológica directa sobre el momento de la ramificación de un linaje filético (que aportan pruebas convincentes sobre un evento real de cladogénesis), a los eventos de especiación se los identifica en paleontología sólo gracias a cambios morfológicos importantes. La asociación de tales eventos de especiación con cambios fenotípicos resulta entonces inevitable, a pesar de que bien podría tratarse de algo más aparente que real. Autores como Hoffman ${ }^{58}$ argumentan, además, que los hiatos estratigráficos con mucha frecuencia guardan una correlación estadística con la evolución fenotípica puntuada. Debido a todo esto, los ejemplos que se han aportado de evolución mediante equilibrio puntuado generalmente son menos sólidos que aquellos que se tienen sobre evolución gradual.

Más interesante todavía resulta la evidencia paleontológica de evolución fenotípica intermedia entre los patrones gradual y puntuado. En algunos casos, la tasa de evolución fenotípica sufrió una aceleración considerable sin que hubiera ninguna relación detectable de algún evento de ramificación de los linajes filéticos. En otros casos, la especiación tuvo lugar relativamente rápido pero, ciertamente, de manera gradual en una escala evolutiva del tiempo. Éste es el caso de evolución por equili-

\footnotetext{
57 HofFMAn (1992); RUSE (1992).

58 Hoffman, A. (1982), «Punctuated versus gradual mode of evolution: a reconsideration», Evol. Biol., 15, pp. 411-436; (1992).
} 
brio puntuado reconocido como el más sólido, el de los moluscos de agua dulce del Lago Turkana - descrito en la primera parte de este trabajo-, donde el propio Williamson ${ }^{59}$ concluye que la especiación tuvo lugar en alrededor de cincuenta mil años (¿a partir de cuánto tiempo o de cuántas generaciones ya no deberíamos considerar la evolución como gradual?).

Este mismo artículo de Williamson ha sido objeto de serios cuestionamientos acerca de la validez de las interpretaciones inferidas de su base empírica, los cuales ilustran nuestro argumento sobre la gran dificultad en obtener evidencias irrefutables a partir de los datos paleontológicos. Sarah Samadi, Patrice David y Philippe Jarne ${ }^{60}$ decidieron utilizar los mismos parámetros empleados por Williamson — es decir, los diseñados por David Raup ${ }^{61}$ para el análisis geométrico de la morfología de las conchas-, en poblaciones naturales de Melanoides tuberculata, uno de los principales especímenes estudiados entre los fósiles del lago Turkana. Dado que M. tuberculata no se ha extinguido, y que hoy en día se le encuentra en casi todos los trópicos, Samadi et. al. pudieron trabajar con las variaciones en las conchas de numerosos ejemplares vivos de diferentes poblaciones. Su objetivo era averiguar si, a partir de los mismos parámetros morfológicos empleados por Williamson, era posible discernir las variaciones de origen genético de las no genéticas. Dado que, por tratarse de especímenes vivos, los resultados morfológicos podían contrastarse con los datos genéticos - lo que es imposible en el caso de los especímenes fósiles-, podrían saberse si el empleo exclusivo de los parámetros de la concha permitía hacer inferencias válidas sobre eventos de especiación. Y los resultados fueron que, en todos los casos estudiados, no era posible saber a partir de la aplicación exclusiva de herramientas morfométricas, si los ejemplares estudiados estaban separados o no por grandes distancias genéticas. Muchos casos de dimorfismo sexual y de plasticidad fenotípica resultaban fácilmente confundibles con casos de especieción si no se contaba con información adicional.

Un último problema para evaluar qué patrón evolutivo es más común, es simplemente que los dos bandos en controversia no han logrado ponerse de acuerdo sobre la forma de estimar cuantitativamente las frecuencias relativas de la evolución fenotípica gradual y de la estasis prolongada. Pero este asunto no es pueril y no será fácil de resolver, ya que es muy difícil trazar adecuadamente una muestra aleatoria de linajes filéticos, a lo largo de la diversidad taxonómica y de los diversos espectros ambientales, en la que los patrones de la evolución fenotípica puedan ser determinados empí-

59 Williamson (1981), p. 441.

60 SAMADI, S.; DAVID, P.; JARNE, P. (2000), «Variation of shell shape in the clonal snail Melanoides tuberculata and its consequences for the interpretation of fossil series», Evolution, 54, pp. 492-502.

61 RAUP, D. (1966), «Geometric analysis of shell coiling: general problems», Journal of Paleontology, 40, pp. 1178-1190. 
ricamente. Hasta ahora los intentos más serios al respecto los han llevado a cabo los puntuacionistas: Cheetham ${ }^{62}$ con un estudio sobre Metrarabdotos (un género de briozoarios marinos) y sobre todo Stanley y Yang ${ }^{63}$, quienes analizaron un grupo de fósiles de bivalvos originarios de la región oeste del Atlántico. Estudiaron 24 variables (normalizadas para el tamaño de la concha) en 19 linajes diferentes, para un total de más de 43,000 mediciones, siguiendo un exhaustivo método de muestreo que abarcó a todas las especies dentro de cuatro taxones de bivalvos (Lucinidae, Tellinacea, Veneridae y Arcticacea), y todo ello dentro de un rango de tiempo de más de 4 millones de años (del Plioceno temprano al reciente). Stanley y Yang también analizaron las variaciones morfológicas de la concha en poblaciones naturales actuales - ya que de las 19 especies estudiadas 12 existen todavía, y en otros 4 casos existen lo que probablemente sean sus descendientes inmediatos-, con lo cual obtuvieron sus estándares de comparación para los especímenes fósiles, además de que lograron establecer una similitud entre las variaciones geográficas de poblaciones actuales con las variaciones en el tiempo presentadas a lo largo de los 4 millones de años estudiados.

Sin embargo, y a pesar de lo exhaustivo de estos estudios, sus resultados no han sido aceptados por la contraparte: Hoffman ${ }^{64}$ alega que sus muestras - si bien son un primer paso en la dirección corrrecta-, se han concentrado en un solo medio ambiente, una sola área geográfica y muy pocos grupos orgánicos (todos los taxones pertenecen a los moluscos), conocidos previamente por ser particularmente favorables para mostrar patrones puntuados de cambio fenotípico. De manera análoga, Stanley ${ }^{65}$ acusa a los gradualistas de asumir que especies fósiles distintas pertenecen a un mismo linaje filético, con tan pocos fósiles intermedios que no es posible demostrar que hubo un cambio morfológico gradual dentro de un solo linaje y además pasando por alto que, con frecuencia, se presentan traslapes entre ellas.

Así pues, en tanto no se pongan de acuerdo ambos bandos de la controversia en un mismo grupo de normas sobre la valoración de los datos fósiles y la forma de estimar cuantitativamente las frecuencias relativas de la evolución fenotípica gradual y puntuada, de poco servirá que sigan acumulándose estudios de campo a favor y en contra del equilibrio puntuado, ya que su valor no será reconocido por la contraparte.

\footnotetext{
62 Cheetham (1986).

63 Stanley, S. M.; YANG, X. (1987), «Approximate evolutionary stasis for bivalve morphology over millions of years: a multivariate, multilineage study», Paleobiology, 13, pp. 113-139.

64 HOFFMAN (1992).

65 Stanley, S. M. (1992), «The Empirical Case for the Punctuational Model of Evolution», en Somit, A.; Peterson, S. (ed.), The Dynamics of Evolution. The Punctuated Equilibrium Debate in the Natural and Social Sciences, Ithaca, Cornell University Press, pp. 85-102.
} 


\section{CONCLUSIONES}

Hemos procurado mostrar qué factores favorecieron la transformación del equilibrio puntuado, desde una fase radical que pretendía fundar una nueva teoría general de la evolución, hasta una teoría de aplicación limitada. Los factores que identificamos como los principales fueron el repliegue de Gould y Eldredge a posiciones menos extremas, la incapacidad del puntuacionismo para hacer mella en el núcleo firme de la síntesis moderna, la inclusión dentro del cinturón protector de la síntesis moderna de los conceptos de estasis y de la evolución mediante tasas variables e intermitentes de cambio fenotípico, aunado todo esto a las dificultades empíricas para validar qué mecanismo es más común, si el gradualismo de la anagénesis o una evolución intermitente que coincide con eventos de cladogénesis. La suma de estas razones explican, a nuestro parecer, que el equilibrio puntuado haya ido deslizándose, poco a poco, hacia una teoría de rango medio.

Ahora bien, ¿qué podemos esperar para el futuro? ¿Podemos esperar que esta teoría abandone su estatus actual e intente nuevamente poner en jaque a la síntesis moderna? Una posibilidad para que esto ocurra sería que, en el futuro, se descubra una nueva técnica que permita extraer mucho más evidencia pertinente a partir del estudio del registro fósil, o bien, que se encuentren nuevas formaciones inusualmente completas y bien representadas, que aporten una mayor y mejor evidencia empírica a favor del equilibrio puntuado, más allá de toda duda.

Sin embargo, coincidimos con Ruse ${ }^{66}$ y con Hoffman ${ }^{67}$ en creer que es poco probable que, al final, sea la evidencia empírica la que vaya a resolver definitivamente la controversia entre el equilibrio puntuado y la síntesis moderna. Es muy difícil que la evidencia paleontológica llegue a ser tan contundente como para descartar cualquiera de los dos mecanismos evolutivos, y consideramos que es más probable que ambas teorías sigan coexistiendo en el futuro. En el campo de la macroevolución seguramente continuará imperando la síntesis moderna como la teoría principal y, sin que afecte su núcleo firme, seguirá presente, como una teoría de rango medio, el equilibrio puntuado.

66 RUSE (1992).

67 HOFFMAN (1992) 
